\title{
Dependence of electron states density of intercalated layer crystal on the intercalant concentration
}

\author{
N.K.Tovstyuk ${ }^{1}$, B.A.Lukiyanets ${ }^{2}$ \\ 1 Ivan Franko Lviv National University \\ 50 Dragomanov Str., 79005 Lviv, Ukraine \\ 2 National University "Lvivska Politekhnika" \\ 12 Bandera Str., Lviv, 79013, Ukraine
}

Received June 11, 2002

\begin{abstract}
Electron states density $\rho(\omega)$ depending on the intercalant atoms concentration within the framework of a virtual crystal is investigated. An essential change of $\rho(\omega)$ in the vicinity of the band bottom of the initial layer crystal at certain energy parameters of a crystal matrix, intercalant and their mutual mixing is obtained. Such a change can manifest itself specifically in optical properties in the region of fundamental absorption.
\end{abstract}

Key words: intercalation, host-guest interaction, electron states density

PACS: $71.20 .-b$

\section{Introduction}

A promising possibility to practically use a high energy density store, stimulates a great interest to the phenomena that permit their realization [1]. Intercalation, as one of the ways of a certain crystal matrix-intercalant system (or in other words host-guest) formation, seems to be very attractive from the point of view of both theoretical and experimental or technological approaches [2]. Investigations of the electron states density $\rho(\omega)$ of a host-guest system is essential for the prediction and understanding of thermodynamic advantages of intercalation-deintercalation processes. The aim of this paper is to study the electron states density $\rho(\omega)$, depending on intercalant concentration $p$ and microscopic parameters of the electron structure, both host and guest subsystems as well as the interaction between them. 


\section{Model}

Calculation of electron states density $\rho(\omega)$ has never been a trivial problem. In this paper, we shall start from the model dispersion law, reflecting the peculiarities of chemical bonds in the layer crystal. It is known [3] that the electron states density $\rho(\omega)$, corresponding to this dispersion law is different from the isotropic or anisotropic semiconductor ones. Particularly, it possesses non-analyticity in the point of $2 \frac{1}{2}$ type Lifshitz transition, which characterizes the transition between the open and the closed isoenergetic surfaces. At a real concentration of free carriers in the layer crystals, the chemical potential can lie in the vicinity of the energy of such a transition.

Electron states density $\rho(\omega)$ of the layer crystal is distorted by the impurities insertion at intercalation. Especially it was demonstrated in the problem for a layer crystal with a single impurity [4]. Let's consider a many impurity problem in the same system. The investigation will be carried out within the framework of the virtual crystal model with the assumption of equiprobable population of every cell by intercalant.

Hamiltonian of the system in the site representation has the form

$$
\begin{aligned}
H= & \sum_{\vec{n}, \vec{n}^{\prime}} t\left(\vec{n}, \vec{n}^{\prime}\right) c_{\vec{n}}^{+} c_{\vec{n}^{\prime}}+p^{2} \sum_{\vec{n}, \vec{n}^{\prime}} l\left(\vec{n}, \vec{n}^{\prime}\right) a_{\vec{n}}^{+} a_{\vec{n}^{\prime}}+p \sum_{\vec{n}} l_{0} a_{\vec{n}}^{+} a_{\vec{n}} \\
& +\left[p \sum_{\vec{n}} W_{0} a_{\vec{n}}^{+} c_{\vec{n}}+\text { h.c. }\right]+\left[p \sum_{\vec{n}, \vec{n}^{\prime}} W\left(\vec{n}, \vec{n}^{\prime}\right) a_{\vec{n}}^{+} c_{\vec{n}^{\prime}}+\text { h.c. }\right] .
\end{aligned}
$$

Here, the first term describes electron mixing on the $\vec{n}^{\prime}, \vec{n}$ host sites $\left(c_{\vec{n}^{\prime}}, c_{\vec{n}}^{+}\right.$are Fermi operators of annihilation and creation, respectively); the second and the third ones are an analogous mixing in a guest subsystem $\left(a_{\vec{n}^{\prime}}, a_{\vec{n}}^{+}\right.$are Fermi operators of annihilation and creation, respectively). The remaining terms describe an electron host-guest mixing in the same and in the nearest cells, respectively. Probability of the cell population by intercalant $p$, generally depends on $\vec{n}$. For a virtual crystal, $p=N_{0} / N$ is constant ( $N_{0}$ is a quantity of intercalants, $N$ is a quantity of cells in the layer crystal). Therefore

$$
t\left(\vec{n}, \vec{n}^{\prime}\right)=t\left(\vec{n}-\vec{n}^{\prime}\right), \quad l\left(\vec{n}, \vec{n}^{\prime}\right)=l\left(\vec{n}-\vec{n}^{\prime}\right), \quad W\left(\vec{n}, \vec{n}^{\prime}\right)=W\left(\vec{n}-\vec{n}^{\prime}\right) .
$$

In momentum representation, (1) takes the form

$$
H=\sum_{\vec{k}} t(\vec{k}) c_{\vec{k}}^{+} c_{\vec{k}}+p^{2} \sum_{\vec{k}} l(\vec{k}) a_{\vec{k}}^{+} a_{\vec{k}}+p \sum_{\vec{k}} W(\vec{k}) c_{\vec{k}}^{+} a_{\vec{k}}+\text { h.c. }
$$

where $W(\vec{k})$ Fourier transformation of $W_{0}+W\left(\vec{n}, \vec{n}^{\prime}\right)$, and

$$
t(\vec{k})=\alpha_{c} k_{\perp}^{2}+\beta_{c}(1-\cos z) ; \quad l(\vec{k})=\alpha_{i} k_{\perp}^{2}+\beta_{i}(1-\cos z)+l_{o} / p .
$$

$t(\vec{k})$ is the crystal lattice dispersion law with bottom in the origin, and the virtual crystal dispersion law $l(\vec{k})$ is counted off $l_{0} / p$, where $l_{0}$ is an intercalant energy level; 
$\alpha_{c, i}=\hbar^{2} / 2 m_{\perp c, i}^{*}$ and $m_{\perp c, i}^{*}$ is the electron effective mass in the plane of host or guest layer, respectively; $\beta_{c}, \beta_{i}$ are the integrals of the electron mixing neighbouring host and guest atoms, respectively, $z \equiv k_{z}$. The lattice constants were chosen to be equal to unity.

A simultaneous choice of an effective mass approximation (for the electron motion in the layer plane) and a tight binding approximation (along c-axis) is justified by the difference in chemical binding along different crystallographic directions in a layer crystal.

Consider a two-time retarded Green's functions [5] $\left\langle\left\langle c_{\vec{k}} \mid c_{\vec{k}}^{+}\right\rangle\right\rangle,\left\langle\left\langle a_{\vec{k}} \mid a_{\vec{k}}^{+}\right\rangle\right\rangle$for host and guest systems. The equations of motion for them and for Green's functions generated by them are of the form

$$
\begin{aligned}
\omega\left\langle\left\langle c_{\vec{k}} \mid c_{\vec{k}^{\prime}}^{+}\right\rangle\right\rangle & =\delta_{\vec{k} \vec{k}^{\prime}}+t(\vec{k})\left\langle\left\langle c_{\vec{k}} \mid c_{\vec{k}^{\prime}}^{+}\right\rangle\right\rangle+p W(\vec{k})\left\langle\left\langle a_{\vec{k}} \mid c_{\vec{k}^{\prime}}^{+}\right\rangle\right\rangle, \\
\omega\left\langle\left\langle a_{\vec{k}} \mid c_{\vec{k}^{\prime}}^{+}\right\rangle\right\rangle & =p^{2} l(\vec{k})\left\langle\left\langle a_{\vec{k}} \mid c_{\vec{k}^{\prime}}^{+}\right\rangle\right\rangle+p W(\vec{k})\left\langle\left\langle c_{\vec{k}} \mid c_{\vec{k}^{\prime}}^{+}\right\rangle\right\rangle, \\
\omega\left\langle\left\langle a_{\vec{k}} \mid a_{\vec{k}^{\prime}}^{+}\right\rangle\right\rangle & =\delta_{\vec{k} \vec{k}^{\prime}}+p^{2} l(\vec{k})\left\langle\left\langle a_{\vec{k}} \mid a_{\vec{k}^{\prime}}^{+}\right\rangle\right\rangle+p W(\vec{k})\left\langle\left\langle c_{\vec{k}} \mid a_{\vec{k}^{\prime}}^{+}\right\rangle\right\rangle, \\
\omega\left\langle\left\langle c_{\vec{k}} \mid a_{\vec{k}^{\prime}}^{+}\right\rangle\right\rangle & =t(\vec{k})\left\langle\left\langle c_{\vec{k}} \mid a_{\vec{k}^{\prime}}^{+}\right\rangle\right\rangle+p W(\vec{k})\left\langle\left\langle a_{\vec{k}} \mid a_{\vec{k}^{\prime}}^{+}\right\rangle\right\rangle,
\end{aligned}
$$

Then (4) gives

$$
\begin{aligned}
\left\langle\left\langle c_{\vec{k}} \mid c_{\vec{k}}^{+}\right\rangle\right\rangle & =\frac{1}{\omega-t(\vec{k})}+\frac{p W(\vec{k})}{\omega-t(\vec{k})}\left\langle\left\langle a_{\vec{k}} \mid c_{\vec{k}}^{+}\right\rangle\right\rangle, \\
\left\langle\left\langle a_{\vec{k}} \mid c_{\vec{k}}^{+}\right\rangle\right\rangle & =\frac{p W(\vec{k})}{\omega-p^{2} l(\vec{k})}\left\langle\left\langle c_{\vec{k}} \mid c_{\vec{k}}^{+}\right\rangle\right\rangle
\end{aligned}
$$

and (5) gives

$$
\begin{aligned}
\left\langle\left\langle a_{\vec{k}} \mid a_{\vec{k}}^{+}\right\rangle\right\rangle & =\frac{1}{\omega-p^{2} l(\vec{k})}+\frac{p W(\vec{k})}{\omega-p^{2} l(\vec{k})}\left\langle\left\langle c_{\vec{k}} \mid a_{\vec{k}}^{+}\right\rangle\right\rangle, \\
\left\langle\left\langle c_{\vec{k}} \mid a_{\vec{k}}^{+}\right\rangle\right\rangle & =\frac{p W(\vec{k})}{\omega-t(\vec{k})}\left\langle\left\langle a_{\vec{k}} \mid a_{\vec{k}}^{+}\right\rangle\right\rangle .
\end{aligned}
$$

As it follows from (7)

$$
\left\langle\left\langle a_{\vec{k}} \mid a_{\vec{k}}^{+}\right\rangle\right\rangle=\frac{\omega-t(\vec{k})}{[\omega-t(\vec{k})] \cdot\left[\omega-p^{2} l(\vec{k})\right]-p^{2} W^{2}(\vec{k})} .
$$

Similarly from (6) it follows

$$
\left\langle\left\langle c_{\vec{k}} \mid c_{\vec{k}}^{+}\right\rangle\right\rangle=\frac{\omega-p^{2} l(\vec{k})}{[\omega-t(\vec{k})] \cdot\left[\omega-p^{2} l(\vec{k})\right]-p^{2} W^{2}(\vec{k})} .
$$

Using a correlation between density of states and a corresponding Green's function [5], the electron states density of the intercalant and the intercalated layer crystal $\rho_{i}(\vec{k}), \rho_{c}(\vec{k})$ are equal, respectively to

$$
\rho_{i}(\vec{k})=-\frac{1}{\pi} \operatorname{Im}\left\langle\left\langle a_{\vec{k}} \mid a_{\vec{k}}^{+}\right\rangle\right\rangle_{\omega \rightarrow \omega+\mathrm{i} \delta}=\frac{\omega-t(\vec{k})}{\omega_{1}-\omega_{2}} \cdot\left\{\delta\left(\omega-\omega_{1}\right)-\delta\left(\omega-\omega_{2}\right)\right\}
$$


and

$$
\rho_{c}(\vec{k})=\frac{\omega-p^{2} l(\vec{k})}{\omega_{1}-\omega_{2}}\left\{\delta\left(\omega-\omega_{1}\right)-\delta\left(\omega-\omega_{2}\right)\right\}
$$

where $\omega_{1}$ and $\omega_{2}$ are poles of Green's function (8) or (9), i.e. solutions of the equation

$$
\omega^{2}-\left[t(\vec{k})+p^{2} l(\vec{k})\right] \cdot \omega+p^{2} l(\vec{k}) \cdot t(\vec{k})-p^{2} W^{2}(\vec{k})=0 .
$$

They are

$$
\omega_{1}=\frac{t(\vec{k})+p^{2} l(\vec{k})}{2}+K ; \quad \omega_{2}=\frac{t(\vec{k})+p^{2} l(\vec{k})}{2}-K
$$

with

$$
2 K=\sqrt{\left[t(\vec{k})-p^{2} l(\vec{k})\right]^{2}+4 p^{2} W^{2}(\vec{k})}
$$

On the assumption of (11), the electron states density of the crystal lattice is

$$
\rho_{c}(\omega)=\sum_{\vec{k}} \rho_{c}(\vec{k})=\frac{2 \pi}{2(2 \pi)^{3}} \int \frac{\omega-p^{2} l(\vec{k})}{2 K}\left[\delta\left(\omega-\omega_{1}\right)-\delta\left(\omega-\omega_{2}\right)\right] \mathrm{d} k_{\perp}^{2} \mathrm{~d} z
$$

Here, cylindrical coordinate system with polar coordinates in the XOY plane is used.

From the equations $\omega-\omega_{1}=0, \omega-\omega_{2}=0$, rewritten in the form

$$
\begin{aligned}
& a-b q_{1}-\sqrt{\left(c q_{1}+d\right)^{2}+p^{2} W^{2}(\vec{k})}=0 ; \\
& a-b q_{2}+\sqrt{\left(c q_{2}+d\right)^{2}+p^{2} W^{2}(\vec{k})}=0 ;
\end{aligned}
$$

$k_{\perp}^{2} \equiv q$, which supply nonzero integrands in $\rho_{i}(\vec{k}), \rho_{c}(\vec{k})$ should be determined. In (16), (17) such indication is accepted

$$
\begin{aligned}
& a=\frac{2 \omega-\left(\beta_{c}+\beta_{i} p^{2}\right)(1-\cos z)-p l_{0}}{2} ; \\
& b=\frac{\alpha_{c}+p^{2} \alpha_{i}}{2} ; \quad c=\frac{\alpha_{c}-p^{2} \alpha_{i}}{2} ; \\
& d=\frac{\left(\beta_{c}-\beta_{i} p^{2}\right)(1-\cos z)-p l_{0}}{2} .
\end{aligned}
$$

We shall assume $W(\vec{k})=W=$ const.

Then, using $\delta$ function property [6]

$$
\delta\left(\omega-\omega_{1}\right)=\delta(f(q))=\sum_{i} \frac{\delta\left(q-q_{i}\right)}{|\partial f / \partial q|_{q=q_{i}}}
$$

$\left(q_{i}\right.$ are solutions of the equations (16) and (17)), (15) may be written in the form

$$
\rho_{c}(\omega)=\frac{1}{2(2 \pi)^{2}} \int_{z_{1}}^{z_{2}} \frac{\omega-p^{2} l\left(q_{1}\right)}{\left|2 b K\left(q_{1}\right)+c\left(c q_{1}+d\right)\right|} \mathrm{d} z-\frac{1}{2(2 \pi)^{2}} \int_{z_{3}}^{z_{4}} \frac{\omega-p^{2} l\left(q_{2}\right)}{\left|2 b K\left(q_{2}\right)-c\left(c q_{2}+d\right)\right|} \mathrm{d} z
$$




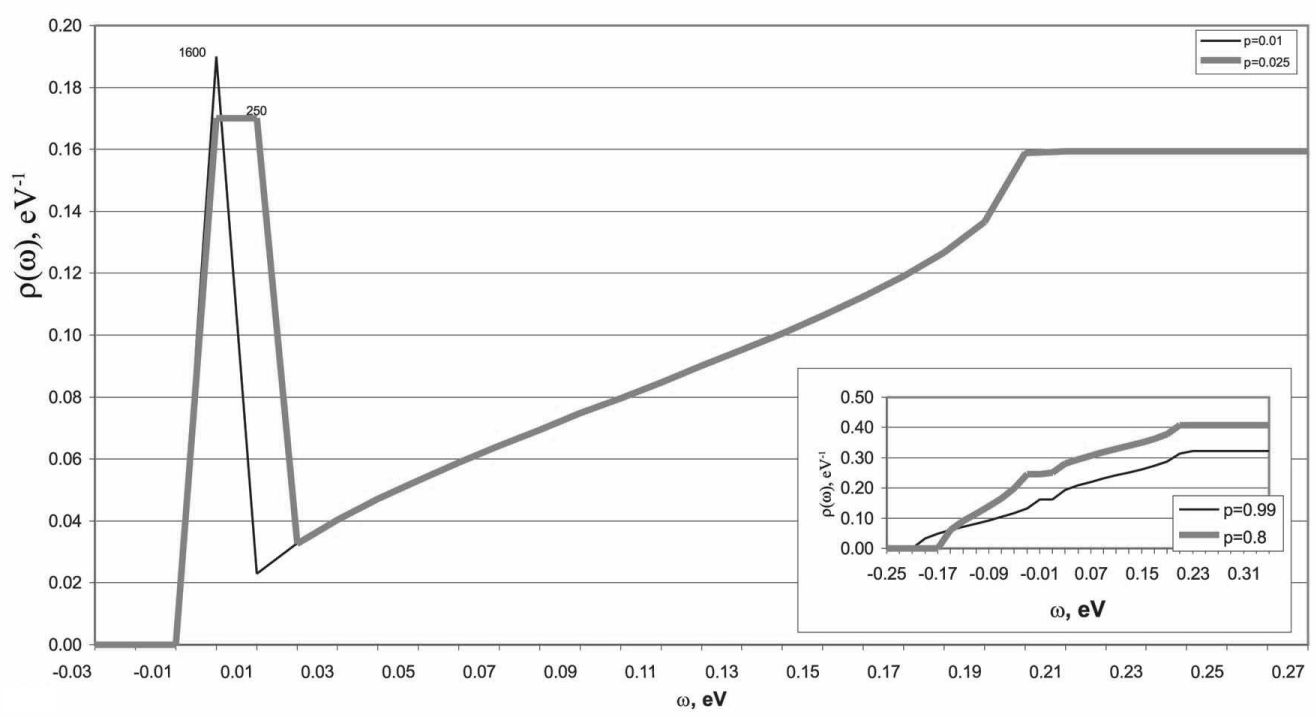

Figure 1. Spectral dependence of total electron states density $\rho(\omega)$ at $l_{0}=$ $-0.20 \mathrm{eV}$ at small $p$ and in insert at great $p$.

and

$$
\rho_{i}(\omega)=\frac{1}{2(2 \pi)^{2}} \int_{z_{1}}^{z_{2}} \frac{\omega-t\left(q_{1}\right)}{\left|2 b K\left(q_{1}\right)+c\left(c q_{1}+d\right)\right|} \mathrm{d} z-\frac{1}{2(2 \pi)^{2}} \int_{z_{3}}^{z_{4}} \frac{\omega-t\left(q_{2}\right)}{\left|2 b K\left(q_{2}\right)-c\left(c q_{2}+d\right)\right|} \mathrm{d} z,
$$

where $K\left(q_{i}\right)$ is $K$ value from (14) at $k_{\perp_{i}}^{2}=q_{i}$; integration regions $z_{i}$ are determined from the condition $(2 \pi)^{2} \geqslant q_{i} \geqslant 0$.

In a special case of $p=0$, it follows $\rho_{i}(\omega)=0$, and electron states density coincides with the same one for an ideal layer crystal:

$$
\rho_{c}(\omega)=\left\{\begin{array}{cc}
\frac{2 \pi}{\alpha_{c}} \arccos \frac{2 \beta_{c}-\omega}{2 \beta_{c}}, & \omega \leqslant 2 \beta_{c} \\
\frac{\pi^{2}}{\alpha_{c}}, & \omega \geqslant 2 \beta_{c}
\end{array} .\right.
$$

\section{Calculation and discussion}

Spectral dependence of electron states density of crystal lattice + intercalant depending on intercalant concentration $p$ was studied. As it has been shown by numerical calculations, peculiarities of the total states density, $\rho(\omega)=\rho_{c}(\omega)+\rho_{i}(\omega)$, are determined mainly by mutual energy host and guest positions. That is why other energy parameters were chosen such as $\alpha_{c}=\alpha_{i}=1.00 \mathrm{eV}, \beta_{c}=\beta_{i}=0.10 \mathrm{eV}$, $W=0.05 \mathrm{eV}$ as it is seen in figure 1 , a sharp peak (1600 is its value) appears in the total electron states density, $\rho(\omega)=\rho_{c}(\omega)+\rho_{i}(\omega)$, near the band bottom of an initial crystal, i.e. $\omega=0$, at $l_{0}=-0.20$ and intercalant concentration $p=0.01$. The greater is $p$, the smaller becomes the peak (see the curve at $p=0.025$ ). Cogent 


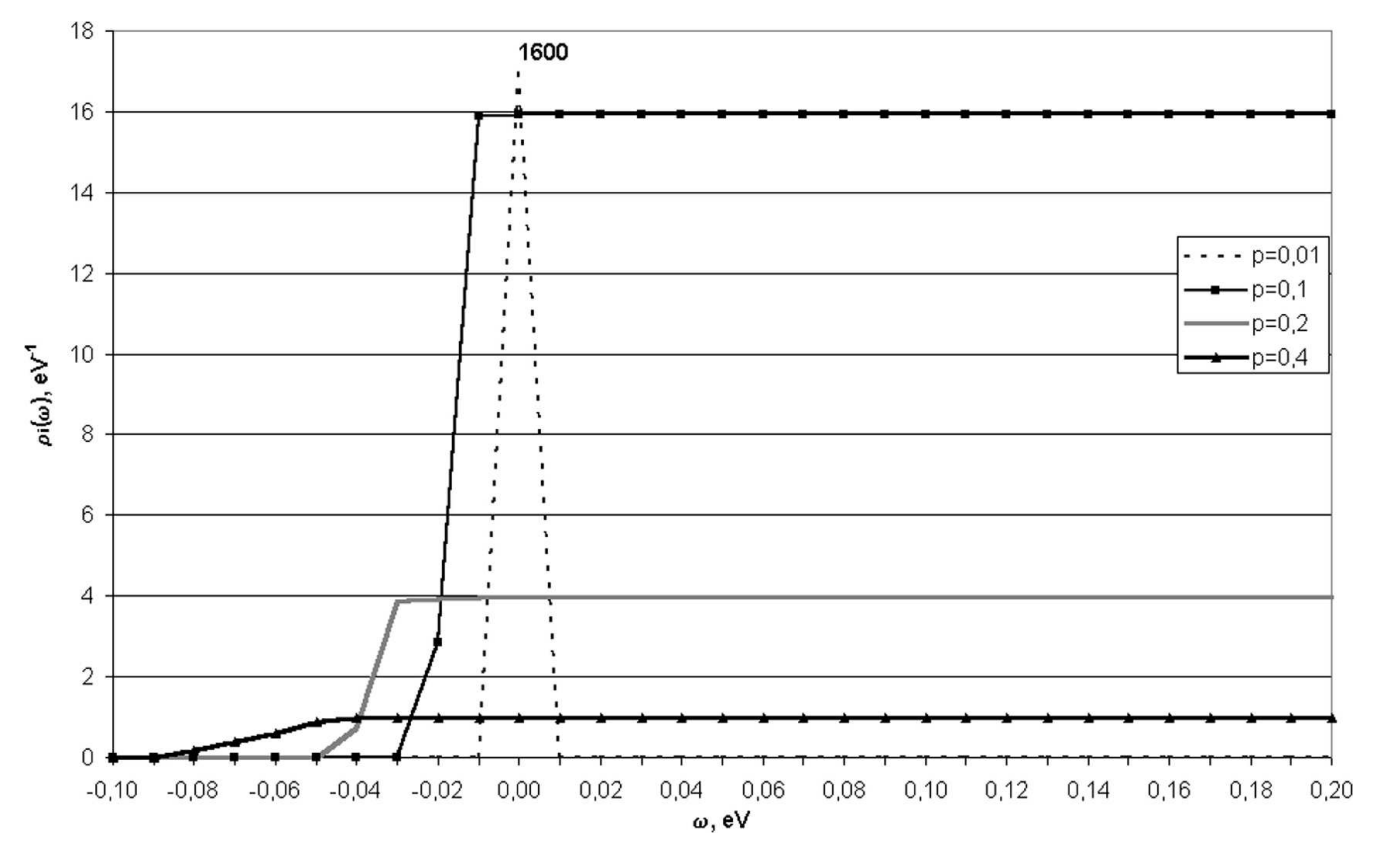

Figure 2. Spectral dependence of intercalant electron states density $\rho_{i}(\omega)$ at $l_{0}=-0.20 \mathrm{eV}$.

argument to this statement is the curves in the insert to figure 1 . It is seen that at $p=0.80$ and $p=0.99$ this peak is absent. From figure 1 it also follows, that at the $p$ increase, $\rho(\omega)$ widen in the direction of $\omega$ decrease. It is obvious, that peculiarities of $\rho(\omega)$ are determined by its components: i) the electron states density of intercalant changed by interaction with host, $\rho_{i}(\omega)$ (figure 2), and ii) electron states density of a layer crystal changed by interaction with intercalant, $\rho_{c}(\omega)$ (figure 3 ). Therefore, let's analyze their dependence on intercalant concentration $p$, and the intercalant energy level position $l_{0}$.

Calculations show that:

- The increase of intercalant concentration, $p$, changes the shape of $\rho_{i}(\omega)$ : at small $p$ density $\rho_{i}(\omega)$ is like a $\delta$-type peak, which is transformed, becoming wider and smaller at the $p$ increase (figure 2 );

- At first glance $\rho_{c}(\omega)$ is similar to electron states density of a pure layer crystal (see $(21)$ ), but at negative $l_{0}$, for instance at $l_{0}=-0.20 \mathrm{eV}, \rho_{c}(\omega)$ changes significantly near $\omega=0$. At small $p$, particularly $p=0.01, \rho_{c}(\omega)$ has additional $\delta$-type peak near $\omega=0$. Its shape depends on $p$ : the smaller is the intercalant concentration the higher is the peak and at $p$ rise, the additional peak shifts in the direction of lower energies and its half-width increases (see figure 3). This peak is caused by host-guest interaction $(W \neq 0)$ : the greater is $W$, the higher is the peak (at $W=0$ additional peak disappears). The smaller are the energy parameters of a guest subsystem $\left(\alpha_{i}, \beta_{i}\right)$ the greater is the contribution of an additional peak.

- Numerical calculations also showed that at $l_{0}=0.20 \mathrm{eV}$, i.e. when the energy 


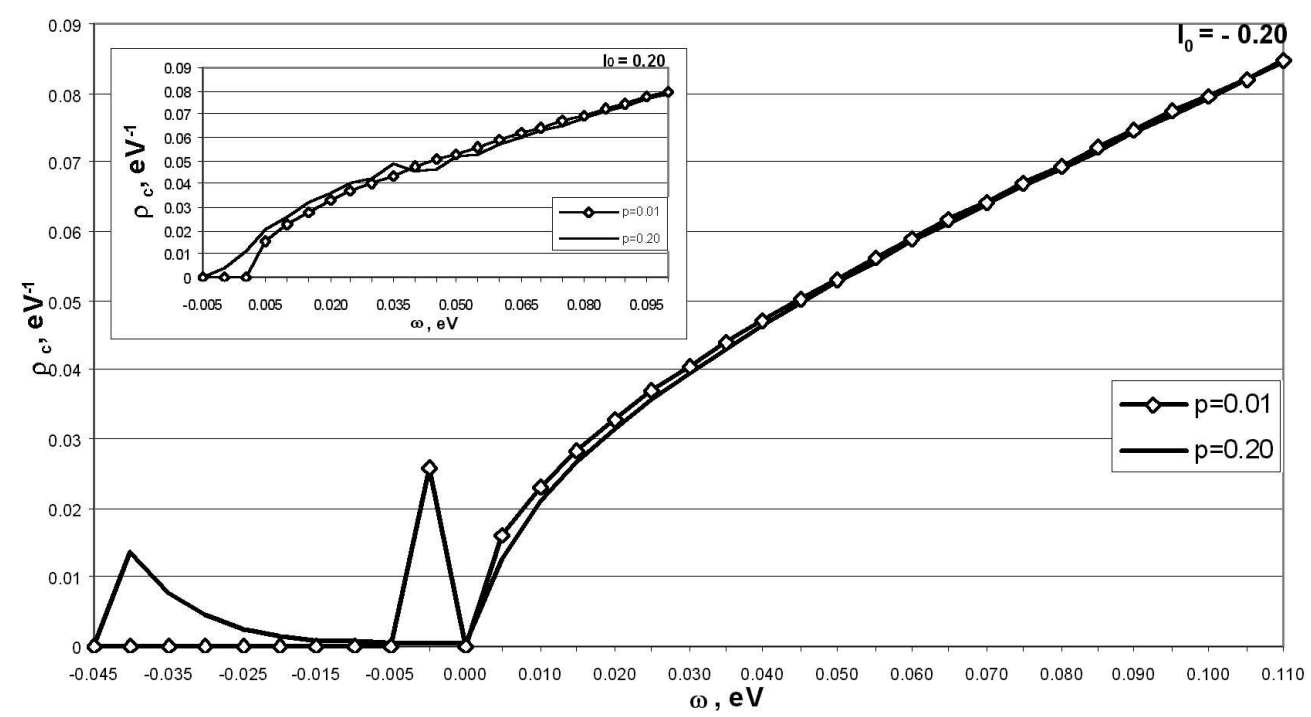

Figure 3. Spectral dependence of electron states density $\rho_{c}(\omega)$ at $l_{0}=-0.20 \mathrm{eV}$, and in insert at $l_{0}=0.20 \mathrm{eV}$.

level of intercalant lies in the conduction band, $\rho_{c}(\omega)$ (see insert to figure 3), differs from $\rho_{c}(\omega)$ obtained at $l_{0}=-0.20 \mathrm{eV}$ : a small $p$ value practically does not change the origin for the host lattice. At the $p$ increase its origin shifts to the left from $\omega=0$.

\section{Conclusions}

For the model dispersion law in the vicinity of high symmetric point of Brillouin zone $\vec{k}=0$, density of electron states is found. Hence, the conclusions of this paper are qualitative ones. However, the main result of the presence of intercalant atoms in the layer crystal is the appearance of a sharp increase of states density near the bottom of the conduction band and its slight broadening. Its value depends both on intercalant concentration and on the energy parameters (the value of host-guest and guest-guest electron mixing). Such changes in the spectrum can lead to optical absorption increase in the region of fundamental absorption, and to the extension of photosensitivity spectra of an intercalated layer crystal which can be used in photoaccumulation.

\section{Acknowledgements}

The investigations carried out were financially supported by STCU (Project No. 818). 


\title{
References
}

1. Grygorchak I.I. // Fizyka i khimija tverdoho tila, 2001, No. 1, p. 7-54 (in Ukrainian).

2. Grygorchak I.I., Seredyuk B.O., Tovstyuk K.D., Bakhmatyuk B.P. NATO-ASI Series "New Trends In Intercalation Compounds For Energy Storage". Edited by C.Julien, J.P.Pereira-Ramos and A.Momchilov. Dordrecht, Kluwer, 2002, p. 543-544.

3. Fivaz R.F. // J. Phys. Chem. Solids, 1967, vol. 28, No. 4, p. 839-852.

4. Lukiyanets B.A., Tovstyuk N.K. // Ukrajinskyj fizychnyj zhurnal, 2001, vol. 46, No. 1, p. 100-104 (in Ukrainian).

5. Zubarev D.N. // Uspekhi fiz. nauk, 1960, vol. 71, p. 71-116 (in Russian).

6. Davydov A.A. Quantum Mechanics. Moscow, 1963 (in Russian).

\section{Залежність густини електронних станів інтеркальованого шаруватого кристала від концентрації інтеркалянта}

\author{
Н.К.Товстюк ${ }^{1}$, Б.А.Лукіянець ${ }^{2}$ \\ 1 Львівський національний університет ім. Івана Франка \\ 79005 Львів, вул. Драгоманова, 50 \\ 2 Національний університет "Львівська Політехніка" \\ 79013 Львів, вул. Бандери, 12
}

Отримано 11 червня 2002 р.

В рамках моделі віртуального кристала досліджена залежність густини електронних станів $\rho(\omega)$ від концентрації інтеркалянта. Суттєві зміни $\rho(\omega)$ в околі дна зони вихідного шаруватого кристала отримано в залежності від енергетичних параметрів гратки і інтеркалянта та їх взаємного перемішування. Такі зміни можуть проявлятися, зокрема, в оптичних властивостях в області власного поглинання.

Ключові слова: інтеркаляція, взаємодія господар-гість, густина електронних станів

PACS: $71.20 .-b$ 